\title{
Classifying Red and Healthy Eyes using Deep Learning
}

\author{
Sherry Verma ${ }^{1}$ Latika Singh $^{2}$, Monica Chaudhry ${ }^{3}$ \\ School of Engineering and Technology, Ansal University, Gurgaon, India ${ }^{1,2}$ \\ Sushant School of Health Science Ansal University, Gurgaon, India ${ }^{3}$
}

\begin{abstract}
Eye is one of the most vital organs of human body. Despite being small in size, humans cannot see the life around them without it. Human eye is protected by a thin covering termed as conjunctiva which protects the eye from dust particles. It plays the role of lubricant in the eye which prevents any sort of friction in opening and closing of eye. Broadly there are two kinds of conjunctiva: bulbar and palpebral. The membrane covering the inner portion of eyelids is termed as palpebral conjunctiva and the one covering the outside portion of the eye is called as bulbar conjunctiva (white portion of eye).Due to the dilation of blood vessels the white portion of the eye also termed as sclera becomes red in color. This condition is also termed as hyperemia. The study of this development is vital in diagnosis of various pathologies. It could be result of some trauma, injury or other eye related diseases which needs to be identified for timely treatment. Enormous amount of studies have been done to study the structure and functionality of human eye. This paper highlights the work done so far for measuring the level of redness in the eye using various methodologies ranging from statistical ways to machine learning techniques and proposes a methodology using Matlab and Convolutional neural network to automate this evaluation process.
\end{abstract}

Keywords-Bulbar conjunctiva; hyperemia; convolutional neural network

\section{INTRODUCTION}

Conjunctival Hyperemia results due to the engorgement of blood vessel in the sclera of the eye. Due to this there is accumulation of blood in the vessels which makes them thicker in size in terms of width. The severe the injury, the more engorgement it results into. As the circumference of the blood vessels gets increased, it results into redness of the affected area. Depending upon how serious the trauma is the level of redness varies. It could range from slight red to severe red. Scholars have worked out various ways of measuring the degree of redness. An effective methodology to measure this is important for diagnosis of various other eye related pathologies. The most important factors that help clinicians to classify hyperemia are degree of redness, hue of the colour and location of the vasodilation [1]. The accurate interpretation of bulbar redness can identify various pathologies like morning eye congestion, bacterial conjunctivitis, dry eye [2], trauma due to prolonged use of contact lenses, iritis and other severe infections [1][3]. It is also a well know side effect of glaucoma treatment [4].Because of these symptoms, patients suffering from the glaucoma drugs often discontinue the treatment [5].Timely and correct diagnosis of these conditions can further reduce any damage to the eye and also help in treatment plan. From last two decades researchers have been working to scale the redness level of the eye in the best possible ways. Most of the work is focussed on subjective analysis of bulbar conjunctivitis using methodologies that involved verbal classifications of redness and/or through pictorial representation. Later the concept of image processing was used extensively to remove the subjective dependency in scaling the redness and various automated approach were presented. This study presents the journey of scaling the hyperemia from manual approach to automated approach by various scholars. The objective of this paper is to analyse the work done so far in the clinical assessment of redness. The paper is organised into various sections: Section II briefly highlights the subjective assessment of hyperemia. In Section III detail research in objectively scaling the hyperemia is mentioned. Section IV highlights potential new way of assessing the redness. Results and conclusion are presented in Sections V and VI.

\section{SUBJECTIVE ASSESSMENT OF HYPEREMIA}

Ocular surface assessment is a routine procedure and one of the common methods to do it is subjective observation and description [6]. Assessment of bulbar redness is quite difficult owing to the contrast of repeat assessment among different clinicians over a period of time [7] [8] [9]. Also various internal and external factors make the subjective analysis much unreliable and results varies from observer to observer [10]. Clinical grading can be performed using at least two general approaches. One that involves the brightness of the eye and other that is based on the various features of the vessels of the concerned area. [8] Paul highlighted the features like vessel tortuosity, number of vessels in the area of study and the diameter of the blood vessels to measure bulbar redness. The clinical assessment of bulbar conjunctivitis is subjective in nature [11] and is poorly understood. It is primarily based on grading scales. The challenge in grading is that the grades themselves are poorly described and moreover due to the varying learning abilities, it is not clear how clinicians grade the redness [10] [12]. The early scale developed was photography based McMonnies/ChapmanDavies scale, which graded conjunctival hyperemia on six levels. [13] It was quite useful for clinical application but was restricted to contact lens wearers. The scale established that hyperemia in soft lens wearer is relatively high as compared to hard lens wearer. Further CCLRU standards (cornea and contact lens research unit), which was earlier termed as IER scale (the Institute for Eye Research scale) was developed [14]. This scale highlighted the clinical performance of lenses with respect to short and prolonged use [15] [16]. It graded the bulbar redness on a 5-point photographic scale where 1 stands for very slight, 2 means slight, 3 signifies moderate and 4 represents the condition as severe. The results of this scale lacked in homogeneity, either in terms of different 
illumination conditions or variability of size of the area under display.Later Efron grading scale was developed which graded the severity of hyperemia on the scale of 0 to 4 [17]. But it again was limited for contact lens complications and images taken into consideration were rendered by an artist to highlight severe condition. Moreover it was not efficient in providing a continuous linear quantitative evaluation.

TABLE. I. COMPARATIVE ANALYSIS OF SCALES USED FOR EVALUATION OF BULBAR REDNESS

\begin{tabular}{|c|c|c|c|c|c|}
\hline Scales & $\begin{array}{l}\text { Grading } \\
\text { Levels } \\
\text { Used }\end{array}$ & $\begin{array}{l}\text { Applicatio } \\
\text { n }\end{array}$ & $\begin{array}{l}\text { Limitati } \\
\text { on }\end{array}$ & $\begin{array}{l}\text { Inte } \\
\text { grac }\end{array}$ & olated \\
\hline \multirow{12}{*}{$\begin{array}{l}\text { McMonnies/Ch } \\
\text { apman-Davies } \\
\text { scale }\end{array}$} & \multirow{12}{*}{$\begin{array}{l}\text { Graded } \\
\text { conjuncti } \\
\text { val } \\
\text { hyperemi } \\
\text { a on six } \\
\text { levels }\end{array}$} & \multirow{12}{*}{$\begin{array}{l}\text { Useful for } \\
\text { clinical } \\
\text { application } \\
\text { s. It also } \\
\text { showed } \\
\text { high } \\
\text { degree of } \\
\text { inter-intra } \\
\text { observer } \\
\text { reliability }\end{array}$} & \multirow{12}{*}{$\begin{array}{l}\text { Restricte } \\
\text { d only to } \\
\text { contact } \\
\text { lens } \\
\text { wearer }\end{array}$} & $\begin{array}{l}\text { VB } \\
\mathbf{R}\end{array}$ & MCD \\
\hline & & & & 0 & - \\
\hline & & & & 10 & - \\
\hline & & & & 20 & 1 \\
\hline & & & & 30 & 2 \\
\hline & & & & 40 & 2.8 \\
\hline & & & & 50 & 4 \\
\hline & & & & 60 & 4.8 \\
\hline & & & & 70 & $>5$ \\
\hline & & & & 80 & $>5$ \\
\hline & & & & 90 & $>5$ \\
\hline & & & & 100 & $>5$ \\
\hline \multirow{12}{*}{$\begin{array}{l}\text { Brien Holden } \\
\text { Vision } \\
\text { Institutescales } \\
\text { (CCLRU) }\end{array}$} & \multirow{12}{*}{$\begin{array}{l}\text { Graded } \\
\text { conjuncti } \\
\text { val } \\
\text { hyperemi } \\
\text { a on 5- } \\
\text { point } \\
\text { photogra } \\
\text { phic } \\
\text { scale }\end{array}$} & \multirow{12}{*}{$\begin{array}{l}\text { Helps in } \\
\text { describing } \\
\text { the } \\
\text { severity of } \\
6 \text { contact } \\
\text { lens } \\
\text { complicati } \\
\text { ons }\end{array}$} & \multirow{12}{*}{$\begin{array}{l}\text { Unable } \\
\text { to depict } \\
\text { homogen } \\
\text { eity } \\
\text { between } \\
\text { different } \\
\text { images } \\
\text { of same } \\
\text { conditio } \\
\text { ns. }\end{array}$} & $\begin{array}{l}\text { VB } \\
\mathbf{R}\end{array}$ & $\begin{array}{l}\text { CCLRU/I } \\
\text { ER }\end{array}$ \\
\hline & & & & 0 & $<1$ \\
\hline & & & & 10 & 2.1 \\
\hline & & & & 20 & 2.4 \\
\hline & & & & 30 & 2.8 \\
\hline & & & & 40 & 3.6 \\
\hline & & & & 50 & $>4$ \\
\hline & & & & 60 & $>4$ \\
\hline & & & & 70 & $>4$ \\
\hline & & & & 80 & $>4$ \\
\hline & & & & 90 & $>4$ \\
\hline & & & & 100 & $>4$ \\
\hline \multirow{12}{*}{ Efron } & \multirow{12}{*}{$\begin{array}{l}\text { Graded } \\
\text { conjuncti } \\
\text { val } \\
\text { hyperemi } \\
\text { a on the } \\
\text { scale of } \\
0 \text { to } 4 \text {. It } \\
\text { use } \\
\text { painted/a } \\
\text { rtist } \\
\text { illustrate } \\
\text { d images }\end{array}$} & \multirow{12}{*}{$\begin{array}{l}\text { Helps in } \\
\text { describing } \\
\text { the } \\
\text { severity of } \\
16 \text { contact } \\
\text { lens } \\
\text { complicati } \\
\text { ons }\end{array}$} & \multirow{12}{*}{$\begin{array}{l}\text { Didn't } \\
\text { provide } \\
\text { efficient } \\
\text { continuo } \\
\text { us linear } \\
\text { quantitat } \\
\text { ive } \\
\text { evaluativ } \\
\text { e }\end{array}$} & $\begin{array}{l}\text { VB } \\
\mathbf{R}\end{array}$ & EFRON \\
\hline & & & & 0 & - \\
\hline & & & & 10 & 0.3 \\
\hline & & & & 20 & \begin{tabular}{l|l} 
& 1 \\
\end{tabular} \\
\hline & & & & 30 & 1.5 \\
\hline & & & & 40 & 2 \\
\hline & & & & 50 & 2.3 \\
\hline & & & & 60 & 2.6 \\
\hline & & & & 70 & 3 \\
\hline & & & & 80 & 3.5 \\
\hline & & & & 90 & $>4$ \\
\hline & & & & 100 & $>4$ \\
\hline $\begin{array}{l}\text { Validated bulbar } \\
\text { redness scale }\end{array}$ & $\begin{array}{l}\text { The } \\
\text { position } \\
\text { of image } \\
\text { was } \\
\text { recorded } \\
\text { on a } \\
\text { range of } \\
0-100 \\
\text { scale }\end{array}$ & $\begin{array}{l}\text { Incorporat } \\
\text { ed the } \\
\text { relevance } \\
\text { of } \\
\text { combining } \\
\text { psychophy } \\
\text { sical and } \\
\text { physical } \\
\text { attributes } \\
\text { of a scale }\end{array}$ & $\begin{array}{l}\text { Limitatio } \\
\mathrm{n} \text { in } \\
\text { evaluatin } \\
\mathrm{g} \text { digital } \\
\text { images } \\
\text { in terms } \\
\text { of ease } \\
\text { of use } \\
\text { reprodu } \\
\text { cibility }\end{array}$ & & \\
\hline
\end{tabular}

Later validated bulbar redness scale was developed which mentioned the importance and relevance of combining psychophysical and physical attributes of a scale [18]. Range of $0-100$ is being used in this scale and it is currently available in two versions. VBR [5] and VBR [10], [19] Schulze further deployed cross-calibrated reference grades which helped in comparison of various already established grades. It proved that estimates of redness were higher on scales with shorter range. These studies covered and detected small changes in hyperemia in the affected region and also some of them were conducted on lens wearers. Bulbar redness is in some cases unevenly distributed, so it requires a methodology which can cover the entire ROI (region of interest) and generate reproducible results with automated approach. Table I summarizes the details of all the grades being used by observers to grade bulbar redness. It also shows the conversion chart of these interpolated grade developed by [20]. The table clearly indicates that no specific scale can be regarded as best or least.

\section{OBJECTIVE ASSESSMENT OF HYPEREMIA}

Looking at the limitation of subjective assessment and its drawbacks in producing varying results, made the scholars work towards finding an objective, continuous and automated way of assessing bulbar redness. Image processing and analysis have been explored by scholars from last two decades to avoid the dependency on subjective grading of hyperemia. With the evolution of computer and sophisticated softwares, this approach of automation gained prominence. Initial study [21] in this area resulted in a semi-automated approach which showed that a variation of only $5 \%$ was there when the same portion of the same eye was analyzed at different times. Also it resulted in less than $0.02 \%$ variation of the same negative when subjected to repeated analysis. Later in [22] an algorithm was proposed which performed computer based image analysis using image smoothing and edge detection methods. It could grade minor to moderate degree of hyperemia and showed a correlation coefficient of 0.6. But the photographs with poor sharpness were not well suited for this analysis. Further an automated approach based on image analysis was proposed which took into consideration mean relative redness and blood vessel area ratio of the affected eye [23]. It reduced the dependence on operator but incurred costs in terms of hardware and software.Some proposed techniques later could differentiate slight change in diurnal cycle [24]. Some but not all methods demonstrated high level of correlation with subjective gradings [25]. Also the work took into consideration relatively smaller patch and therefore was different from clinical grading as in clinical grading both nasal as well as temporal area are considered. In [26] an automated estimator was developed for conjunctival hyperemia using multivariate regression technique. The correlation coefficients for nasal side were 0.98 and for temporal side was 0.96 . But the study took into consideration very less features. Later [27] proposed an estimator which established the relationship between clinical grading and quantitative aspects of conjunctiva images. The study focussed on elimination of irreproducibility and inconsistency of the clinical ratings in order to get accurate and consistent results. Though the proposed estimator reliably predicted median clinical grades 
but the results were not linear for higher grades of bulbar redness. Another study [28] proposed the use of image analysis to quantify changes in hyperemia. Techniques like color extraction, edge detection and thresholding were used to have a higher level of repeatability. In [29] another objective method was performed to measure bulbar redness using Spectrascan650 Photometer by Photo Research. This study drew comparisons between objectively measuring hyperemia through photometric method with standard grading techniques. Further [30] establishedobjective grading methods which were sensitive and more reliable in detecting and monitoring ocular surface changes. Regression analysis was used to establish correlation between subjective grades and objective measures.

Later in [31] scientists looking into the tedious and time consuming job of grading hyperemia, proposed an automatic approach for selection of best frame for grading. The results of this automatic approach were repeatable, objective and less time consuming. The frames were selected by taking into consideration features like lightness, non-blurriness, or high contrast.This work was further extended in [32] where concept of artificial neural network was incorporated to grade bulbar redness. The proposed methodology transformed the extracted features into grading scales using radial basis function network [33] and multi-layer perceptron [34]. This study implemented extraction of region of interest from best frame. Later various features were computed from region of interest. Lastly artificial neural network was used to transform these features to specialists grading scales. The study however consideredlimited set of features for evaluation. In [35] machine learning techniques like regression and classification were used and their results were compared in grading the red eye. In another study [36] focus was on considering image processing by implementing image segmentation in the evaluation of redness.

Though the objective assessment of bulbar redness has proved to be less time consuming by reducing the involvement of clinical judgement. Still there is a constant need of features extraction involved, which are further transformed into the grading scales. The objectivity thus still is biased on the features selected. The study presented in this paper automatized the entire process of differentiating healthy eye from red eye by extracting the color intensity from the eye and feeding this extracted image to convolution neural network.

\section{METHODOLOGY}

\section{A. Data Collection}

Twenty eight images of bulbar redness were used and twenty eight images of healthy eyes were used. The images were captured throughthree step slit lamp model number HUVITZ 500N using CANON EOS 1300Dcamera attached to it by vision centre of school of health sciences. The images were imported through the software HIS500N (HUVITZ imaging slit lamp).

\section{B. Color Extraction from Input Image using MATLAB}

The coloured images of red and healthy eyes were acquired from vision centre. The images were then read through imread () function [37] of MATLAB. The function returns the input image in the form of array. The color image is returned in the form of three dimensional matrix. After application of various functions of MATLAB, the white color from these input images was extracted using the image processing toolkit of MATLAB.

\section{Algorithm for colour extraction from images}

1. Read an input image

2. Display the original image

3. Extract the individual red, green and blue color channels

4. Define a threshold value of 200

5. Generate a binary image where each color channel extracted above is more than the threshold value

6. Binary image showing white portion is now extracted

7. Display the binary image

8. Stop

Initial requirement of image processing task is to have a coloured image referred to as RGB image. The RGB format of the image is stored as $m * n * 3$ matrix that defines red, green and blue color component for each pixel. The red, green and blue color channels are extracted from the original image. They are stored as three independent two dimensional matrix for each color channel. Further a threshold value is defined to extract the white portion from all these three two dimensional matrix. The binary image [38] [39] [40] is finally generated where each color channel has value greater than the threshold value. Fig. 1 shows the extracted white portion of the healthy eye.

Fig. 2 shows that in case of severe red eye there is minimal white portion.

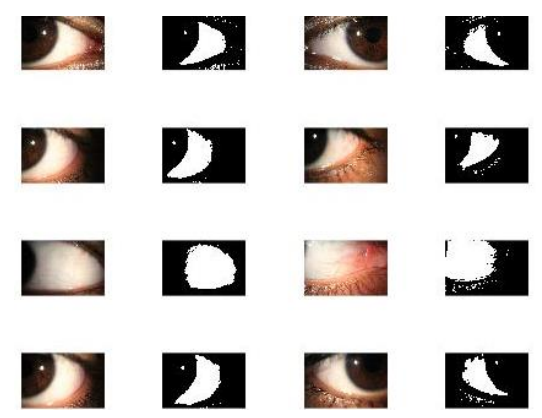

Fig. 1. Extraction of White Color from Healthy Eye.

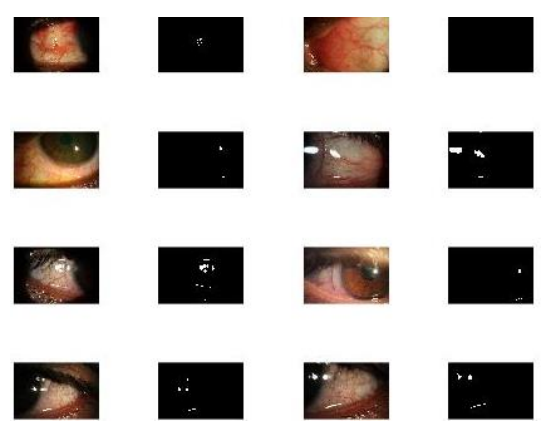

Fig. 2. Extraction of White Color from Red Eye. 


\section{Binary Images fed to Convolutional Neural Network}

1) Convolutional neural network: Convolutional neural network is a special kind of neural network which can work on images ranging from one dimensional, two dimensional to three dimensional. Simple neural network doesn't stand good on feature extraction and feature learning. On the hand CNN works efficiently on excellent feature extraction and learning. The advantage of ConvNet over regular neural network is that it considers every input as image. This consideration helps in creating the architecture in much better way. The neurons of the CNN are arranged in three dimensions $\mathrm{w}^{*} \mathrm{~h} * \mathrm{~d}$, where $\mathrm{w}$ is the width, $h$ is the height and $d$ is the depth. For an RGB image depth is 3 . Each corresponding to red, green and blue color channel.The Convolutional neural network consist of the three main layers. [41] Convolutional layer, pooling layer and fully connected layer. The Convolutional layer is the core of the network that does maximum of the work [42]. The purpose of this layer is to extract high level features from the input image, this is achieved by striding the filter/ kernel over the input image. A kernel is a matrix whose size depends upon the input image being processed. If the image is having multiple channels then the depth of the kernel is same as that of the input image. Convolutional layer doesn't restrict to only one, the network can have many convolutional layer as per need. Further ReLU (rectified linear unit) is applied to the output of convolved layer. Its main purpose is to minimize the computation time [43]. Similar to the convolutional layer the pooling layer main task is to reduce the size of the convolved image. The most popular being the max pooling method [44]. It helps in extracting the dominant features by selecting the maximum value from the portion of image covered by kernel. At the end the output is flattened and fed to the fee forward neural network and backpropagation is applied. After number of iteration the model is able to classify the features.

2) CNN based classifier: We have now a self-curated dataset consisting of extracted "white" portions from human eyes. The goal of this classifier is to classify input image of human eye in two categories, i.e. "Red" or "Healthy". The proposed model start-off by splitting the dataset into training set $(75 \%)$ and testing set $(25 \%)$, followed by augmenting the images in the training set. The image augmentation techniques included rotation, width shifting, height shifting, shear transformation, zoom, and horizontal flipping [45]. The whole dataset was normalized, and the images were resized to a size of $28 \times 28$. For the purpose of training a model, we used Convolutional Neural Networks. A convolutional neural network learns Spatial Dependencies for the representation of images. The convolutional layer makes use of filters, also known as kernels, to form a matrix by sliding the filter over the input image and computing the dot product. This matrix is called a "Convolved Feature Map" or simply "Feature Map". The feature map is directly influenced by Pooling and Strides. Pooling progressively reduces the spatial size of input images. This further reduces the amount of computations. We make use of Max Pooling in the training of our model. Secondly,
Stride refers to the number of pixel shifts that a kernel is supposed to make while extracting features from the image representation. Moving on to the architecture, a Sequential neural network was developed that consisted of the following layers:

- Convolution2D layer with a number of 20 output filters, kernel size of $5 \times 5$, zero padding and input shape of $3 \times 28 \times 28$

- Activation layer of ReLu - Rectified Linear Unit

- Max Pooling layer with a pool size of $2 \times 2$, and $(2 \times 2)$ strides

- The above orientation was repeated again but now with a number of 50 output filters in convolution2D layer.

- Flatten-The output from second pooling layer is flattened into a $1 \mathrm{D}$ array

- A fully connected layer consisting of 500 nodes.

- Another ReLu activation layer

- Finally, the output layer consisting of a single node with Softmax activation function.

The dataset was trained for 100 epochs with a batch size of 32. The model was compiled using "Adam" optimizer [46] [47] with an initial learning rate of 0.001 , further decaying at the rate of 1e-5. The results were quite satisfying. After a total of 100 epochs, we got a training accuracy of $96.88 \%$, with a loss of $11.9 \%$. The validation accuracy had reached $93.33 \%$ with a validation loss of $21.83 \%$. As can be seen, our convolutional neural network performed really well in classifying human eyes as healthy and infected (Red). We propose this model for the noble cause, so that no patient with healthy eyes is to undergo the time-consuming check-up procedure.

\section{RESULTS}

Table II shows test results of the model for healthy eyes. The result includes an input image which was generated after extracting the white portion of the original healthy eye. The output includes the labelled results along with accuracy which we got after feeding the extracted image to our proposed CNN based model. It clearly shows that our model is able to classify well healthy test images passed to as healthy.

Table III shows the output of the model for red eyes. The result includes an input image as test image without label. The output is the correct prediction of the model for red eye with high accuracy. The output images are generated with label as red.

For testing the accuracy of the model a set of 15 images of red as well as healthy eyes each were passed to the model without labels. The model predicted well in both the cases along with labels as red or healthy.

The output accuracy of Table II and Table III are collated in Fig. 3. The figure shows the average accuracy of classifying healthy and red eyes. The chart shows an average accuracy of 94.17 for healthy eyes and 99.99 for red eyes. 
TABLE. II. LABELLED OUTPUT OF HEALTHY EYES WITH ACCURACY

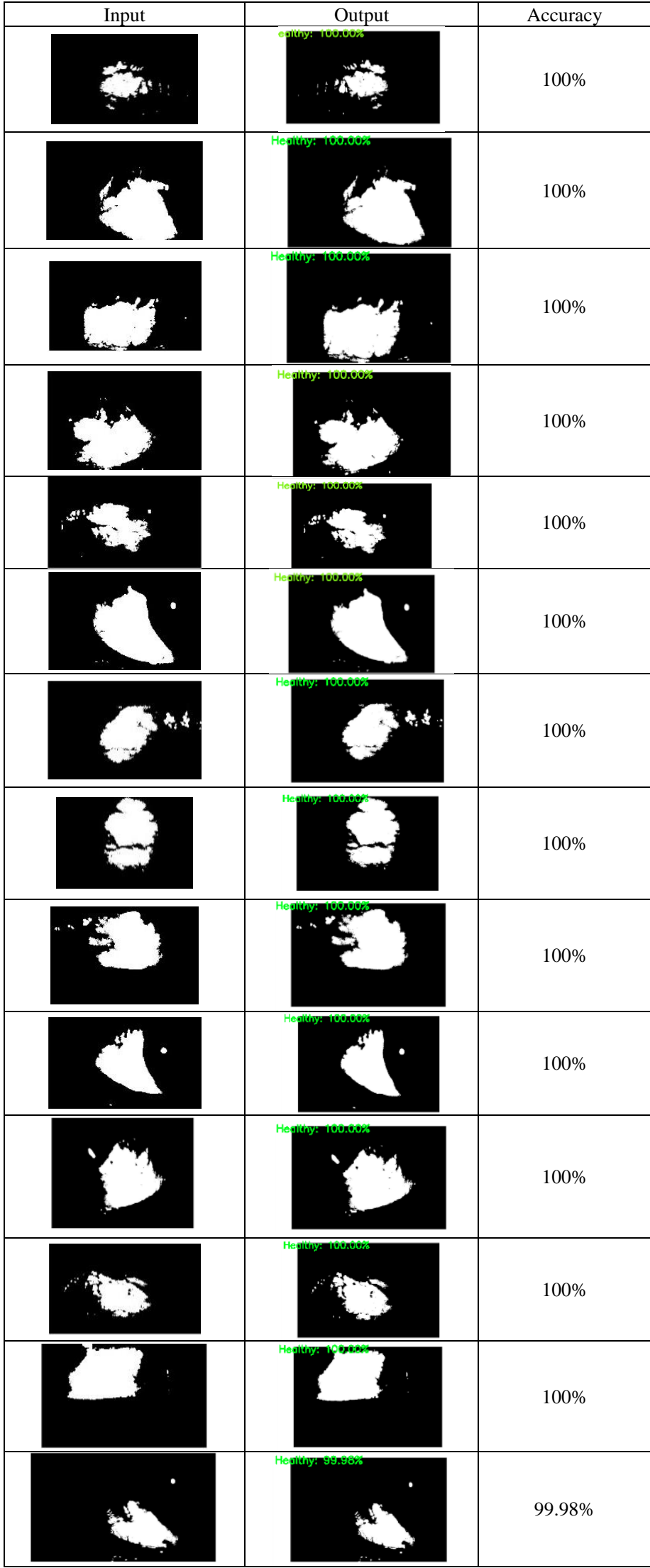

TABLE. III. LABELLED OUTPUT OF RED EYES WITH ACCURACY

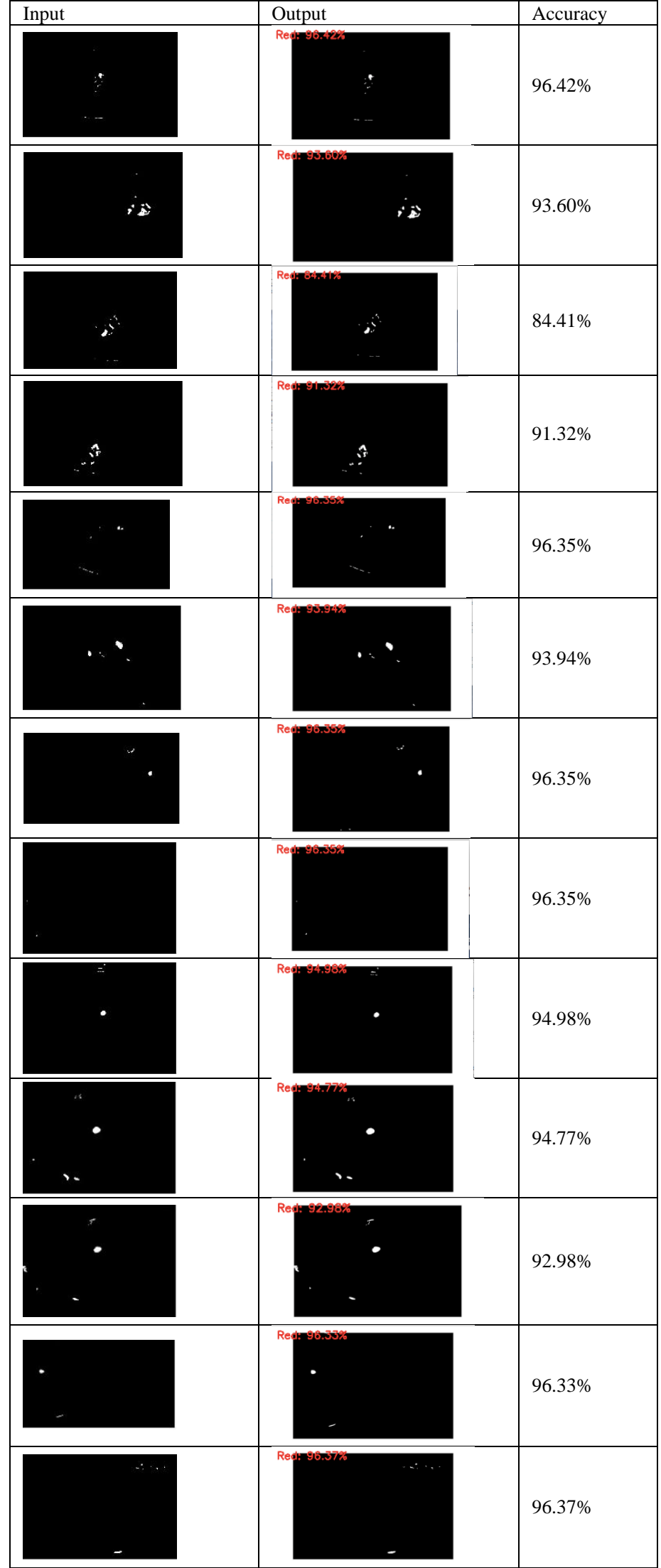




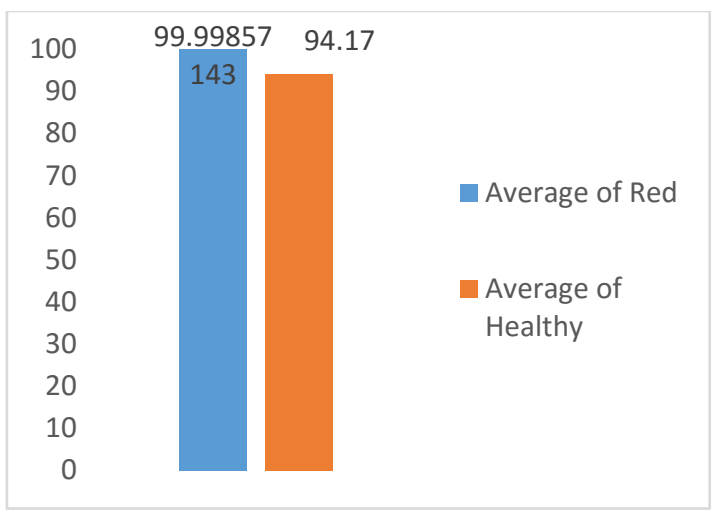

Fig. 3. Average Accuracy of Red and Healthy Eyes.

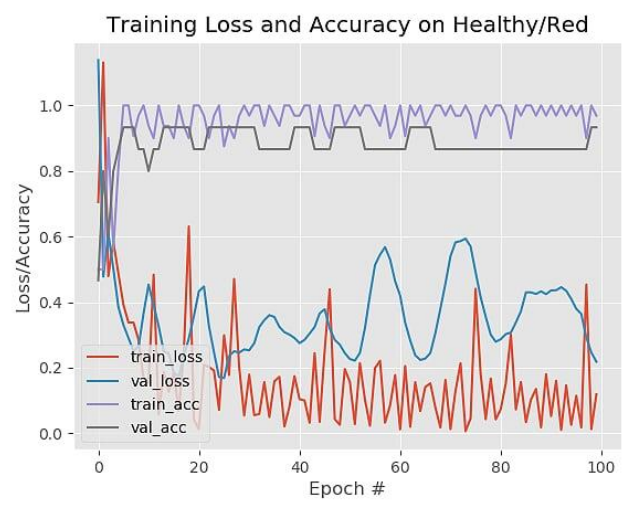

Fig. 4. Loss and Accuracy Plot of Red and Healthy Eyes w.r.t Epochs.

Graph in Fig. 4 shows the validation loss, training loss, validation accuracy and training accuracy of healthy as well as red eyes for 25 epochs, which clearly shows the efficiency of the proposed model.

\section{CONCLUSION}

The main purpose of this study was to develop an effective and efficient CNN based classifier which could distinguish well between a healthy and red human eye in minimum time. The work was accomplished by using MATLAB for extraction of white portion from the eye and then feeding these images to the well-designed convolutional neural network. The CNN based model was coded in Keras, one of the efficient frameworks of Python. The results clearly indicate that our model is accurately classifying between the two types of images. This study will definitely cater to the need of rural India where the ratio of clinicians to the patients is very less. It also highlighted in this paper that neither the subjective method nor the objective method rules out the dependencies on the operators for evaluation and feature extraction. Therefore our proposed model minimizes this dependency by automatically extracting features and classifying the result in no time.

\section{ACKNOWLEDGMENTS}

We would like to thank Sushant School of Health Science, Ansal University, for helping us in providing test images, clinical guidance and expertise.

\section{REFERENCES}

[1] "Abelson M B, Lane K and Maffei C 2010 Code red: The key features of hyperemia. Rev Ophthalmol, 17(4), 9294", www.reviewofophthalmology.com, 2010.

[2] G. Ousler, P. Gomes, D. Welch and M. Abelson, "Methodologies for the Study of Ocular Surface Disease", The Ocular Surface, vol. 3, no. 3, pp. 143-154, 2005. Available: 10.1016/s1542-0124(12)70196-9.

[3] X. Wang, Z. Wu, L. Chen, H. Zheng and H. Yang, "Pixel classification based color image segmentation using quaternion exponent moments", Neural Networks, vol. 74, pp. 1-13, 2016. Available: 10.1016/j.neunet.2015.10.012.

[4] J. Schuman, "Short- and long-term safety of glaucoma drugs", Expert Opinion on Drug Safety, vol. 1, no. 2, pp. 181-194, 2002. Available: 10.1517/14740338.1.2.181.

[5] F. Honrubia, J. Garcia-Sanchez, V. Polo, J. de la Casa and J. Soto, "Conjunctival hyperaemia with the use of latanoprost versus other prostaglandin analogues in patients with ocular hypertension or glaucoma: a meta-analysis of randomised clinical trials", British Journal of Ophthalmology, vol. 93, no. 3, pp. 316-321, 2009. Available: 10.1136/bjo.2007.135111.

[6] J. Rodriguez, P. Johnston, G. Ousler III, M. Abelson and Smith, "Automated grading system for evaluation of ocular redness associated with dry eye", Clinical Ophthalmology, p. 1197, 2013. Available: 10.2147/opth.s39703.

[7] N. Efron, P. Morgan and S. Katsara, "Validation of grading scales for contact lens complications", Ophthalmic and Physiological Optics, vol. 21 , no. 1, pp. 17-29, 2001. Available: 10.1046/j.14751313.1999.00420.x-i1.

[8] P. Fieguth and T. Simpson, "Automated Measurement of Bulbar Redness", Investigative Ophthalmology \& Visual Science, vol. 43, no. 2 , pp. 340-347, 2002.

[9] N. Efron, P. Morgan and R. Jagpal, "The combined influence of knowledge, training and experience when grading contact lens complications", Ophthalmic and Physiological Optics, vol. 23, no. 1, pp. 79-85, 2003. Available: 10.1046/j.1475-1313.2003.00091.x.

[10] W. Zhao, F. Duan, Z. Li, H. Yang, Q. Huang and K. Wu, "Evaluation of regional bulbar redness using an image-based objective method", International journal of ophthalmology, vol. 7, no. 1, p. 71, 2014. Available: 10.3980/j.issn.2222-3959.2014.01.13.

[11] S. Stevens, "On the Theory of Scales of Measurement", Science, vol. 103 , no. 2684 , pp. 677-680, 1946. Available: 10.1126/science.103.2684.677.

[12] T. Chong, T. Simpson and D. Fonn, "The Repeatability of Discrete and Continuous Anterior Segment Grading Scales", Optometry and Vision Science, vol. 77, no. 5, pp. 244-251, 2000. Available: 10.1097/00006324-200005000-00011.

[13] C. McMONNIES and A. CHAPMAN-DAVIES, "Assessment of Conjunctival Hyperemia in Contact Lens Wearers. Part II", Optometry and Vision Science, vol. 64, no. 4, pp. 251-255, 1987. Available: 10.1097/00006324-198704000-00004.

[14] R. TERRY et al., "CCLRU Standards for Success of Daily and Extended Wear Contact Lenses", Optometry and Vision Science, vol. 70, no. 3, pp. 234-243, 1993. Available: 10.1097/00006324-199303000-00011.

[15] A. Phillips and L. Speedwell, Contact Lenses. Oxford: ButterworthHeinemann, 1997, pp. 863-7.

[16] IER. IER grading scales. Institute for Eye Research: Sydney, Australia; 2007.

[17] N. Efron, "Clinical application of grading scales for contact lens complications", Optician, vol. 213, no. 5604, pp. 26-34, 2019.

[18] M. SCHULZE, D. JONES and T. SIMPSON, "The Development of Validated Bulbar Redness Grading Scales", Optometry and Vision Science, vol. 84, no. 10, pp. 976-983, 2007. Available: 10.1097/opx.0b013e318157ac9e.

[19] M. Schulze, N. Hutchings and T. Simpson, "Grading Bulbar Redness Using Cross-Calibrated Clinical Grading Scales", Investigative Opthalmology \& Visual Science, vol. 52, no. 8, p. 5812, 2011. Available: 10.1167/iovs.10-7006. 
[20] M. Schulze, N. Hutchings and T. Simpson, "The Conversion of Bulbar Redness Grades Using Psychophysical Scaling", Optometry and Vision Science, vol. 87, no. 3, pp. 159-167, 2010. Available: 10.1097/opx.0b013e3181ce07f1.

[21] P. Chen, S. Kovalcheck and B. Zweifach, "Analysis of microvascular network in bulbar conjunctiva by image processing", International journal of microcirculation, clinical and experimental, vol. 6, no. 3, pp. 245-255, 1987.

[22] J. Villumsen, J. Ringquist and A. Alm, "Image analysis of conjunctival hyperemia", Acta Ophthalmologica, vol. 69, no. 4, pp. 536-539, 2009. Available: 10.1111/j.1755-3768.1991.tb02036.x.

[23] F. Willingham, K. Cohen, J. Coggins, N. Tripoli, J. Ogle and G. Goldstein, "Automatic quantitative measurement of ocular hyperemia", Current Eye Research, vol. 14, no. 12, pp. 1101-1108, 1995. Available: 10.3109/02713689508995816.

[24] M. GUILLON and D. SHAH, "Objective Measurement of Contact LensInduced Conjunctival Redness", Optometry and Vision Science, vol. 73, no. 9, pp. 595-605, 1996. Available: 10.1097/00006324-19960900000006.

[25] E. Papas, "Key factors in the subjective and objective assessment of conjunctival erythema", Investigative ophthalmology \& visual science, vol. 41, no. 3, pp. 687-691, 2000.

[26] J. Cullen, S. Pounder, K. Whitear and P. Fieguth, "Analysis of CornealImages for Assessing Contact Lens Trauma", in International Conferenceon Image Processing, Vancouver, British Columbia, Canada, 2000.

[27] P. Fieguth and T. Simpson, "Automated measurement of bulbar redness", Investigative ophthalmology \& visual science, vol. 43 , no. 2 , pp. 340-347, 2002.

[28] J. Wolffsohn and C. Purslow, "Clinical monitoring of ocular physiology using digital image analysis", Contact Lens and Anterior Eye, vol. 26, no. 1, pp. 27-35, 2003. Available: 10.1016/s1367-0484(02)00062-0.

[29] L. Sorbara, T. Simpson, S. Duench, M. Schulze and D. Fonn, "Comparison of an objective method of measuring bulbar redness to the use of traditional grading scales", Contact Lens and Anterior Eye, vol. 30, no. 1, pp. 53-59, 2007. Available: 10.1016/j.clae.2006.12.003.

[30] R. Peterson and J. Wolffsohn, "Objective Grading of The Anterior Eye", Optometry and Vision Science, vol. 86, no. 3, pp. 273-278, 2009. Available: 10.1097/opx.0b013e3181981976.

[31] L. Brea, N. Rodríguez, A. González, H. Verdeal and E. Vilar, "Automatic selection of video frames for hyperemia grading", in International Conference on Computer Aided Systems Theory, 2015.

[32] L. Brea, N. Rodríguez, H. Pena-Verdeal and E. Yebra-Pimentel Vilar, "A novel framework for hyperemia grading based on artificial neural networks", in International Work-Conference on Artificial Neural Networks, 2015.

[33] J. Park and I. Sandberg, "Approximation and Radial-Basis-Function Networks", Neural Computation, vol. 5, no. 2, pp. 305-316, 1993. Available: $10.1162 /$ neco.1993.5.2.305.
[34] E. Baum, "On the capabilities of multilayer perceptrons", Journal of Complexity, vol. 4, no. 3, pp. 193-215, 1988. Available: 10.1016/0885064x(88)90020-9.

[35] L. Sánchez Brea, N. Barreira Rodríguez, A. Mosquera González, H. Pena-Verdeal and E. Yebra-Pimentel Vilar, "Comparing machine learning techniques in a hyperemia grading framework", in 8th International Conference on Agents and Artificial Intelligence, 2016.

[36] M. Mohd Adnan, A. Mohd Zain, H. Haron, R. Alwee, M. Zulfaezal Che Azemin and A. Osman Ibrahim, "Eye Redness Image Processing Techniques", Journal of Physics: Conference Series, vol. 892, p. 012019, 2017. Available: 10.1088/1742-6596/892/1/012019.

[37] A. McAndrew, "An introduction to digital image processing with matlab notes for scm2511 image processing", School of Computer Science and Mathematics, Victoria University of Technology, vol. 264, no. 1, pp. 1264, 2004.

[38] L. Vincent, "Morphological grayscale reconstruction in image analysis: applications and efficient algorithms", IEEE Transactions on Image Processing, vol. 2, no. 2, pp. 176-201, 1993. Available: 10.1109/83.217222.

[39] Y. Solihin and C. Leedham, "The multi-stage approach to grey-scale image thresholding for specific applications", Nanyang Technological University, School of Computer Engineering, Nanyang Avenue, Republic of Singapore, 2000, pp. 1-27.

[40] R. C. Gonzalez, R. E. Woods and S. L. Eddins, Digital Image Processing Using MATLAB. Pearson Education, 2004.

[41] Y. LeCun, K. Kavukcuoglu and C. Farabet, "Convolutional networks and applications invision", in IEEE International Symposium on Circuits and Systems, 2010.

[42] Y. LeCun and Y. Bengio, "Convolutional networks for images, speech, and time series", The handbook of brain theory and neural networks, vol. 3361, no. 10, 1995.

[43] V. Nair and G. E. Hinton, "Rectified linear units improve restricted Boltzmann machines", in 27th International Conference on Machine Learning, 2010.

[44] Y. Lecun, L. Bottou, Y. Bengio and P. Haffner, "Gradient-based learning applied to document recognition", Proceedings of the IEEE, vol. 86, no. 11, pp. 2278-2324, 1998. Available: 10.1109/5.726791.

[45] P. Y. Simard, D. Steinkraus and J. C. Platt, "Best practices for convolutional neural networks applied to visual document analysis", in Seventh International Conference on Document Analysis and Recognition, 2003, pp. 958-962.

[46] D. P. Kingma and J. Lei Ba, "Adam : A method for stochastic optimization", in International Conference for Learning Representations, San Diego, 2014.

[47] J. Gu et al., "Recent advances in convolutional neural networks", Pattern Recognition, vol. 77, pp. 354-377, 2018. Available: 10.1016/j.patcog.2017.10.013. 